\title{
HEAT AND MASS TRANSFER ACROSS TURBULENT GAS-LIQUID INTERFACES
}

\author{
Sanjoy Banerjee \\ Distinguished Professor of Chemical Engineering Director of the CUNY Energy Institute, The City College \\ of New York
}

\begin{abstract}
Scalar exchange between turbulent gas and liquid streams separated by deformable and breaking interfaces is of central importance in many environmental and industrial processes. For example, ocean uptake of greenhouse gases is largely governed by liquid-side mass transfer coefficients at the atmosphere water surfaces. Because measurements and analysis of fluid motion and scalar fields very close to deforming interfaces is difficult, our understanding of the governing phenomena is still poor compared to what we know about transport processes in solid-fluid boundary layers. We will discuss recent developments in direct numerical simulations and particle imaging velocimetry that have elucidated turbulence behavior at wavy gas-liquid surfaces. The results indicate that the surface renewal and surface divergence models, which are commonly used to parameterize liquid side controlled transfer rates are inadequate when interfaces micro-break. Theoretical approaches, which combine elements of the surface renewal and divergence models, will be discussed and compared with recent experimental data. The range of applicability of existing surface renewal small models will be discussed as well in the context of the new data.
\end{abstract}

